\title{
A GENERALIZATION OF A PROPERTY OF AN ACNODAL CUBIC CURVE
}

BY HAROLD HILTON

1. Introduction. We refer to the following property.

If in a plane cubic curve there is inscribed a real triangle $A B C$ such that $B C, C A, A B$ touch the curve at $C, A, B$, then the cubic can be projected by a real projection so as to have trigonal symmetry, i.e., it can be brought to self-coincidence by rotating it through $2 \pi / 3$ about a point. If, in particular, the cubic is unicursal (rational), it must be acnodal.*

The generalization suggested is that of any unicursal curve in which a triangle $A B C$ is inscribed, so that $A, B, C$ are each given by a single value of a parameter in terms of which the coordinates of any point of the curve are rationally expressed, while the intersections of $B C, C A, A B$ with the curve lie respectively $p$ at $C$ and $q$ at $B, p$ at $A$ and $q$ at $C, p$ at $B$ and $q$ at $A$. We shall investigate the properties of such curves.

Take the parameters of $A, B, C$ as $0, \infty, 1 . \dagger$ Then choosing suitable homogeneous coordinates, we have evidently

$$
x: y: z=(t-1)^{p}:(-t)^{p}(t-1)^{q}:(-t)^{q} .
$$

We shall find it convenient to use a quantity $\epsilon$ defined by

$$
\epsilon \equiv p^{2}-p q+q^{2}
$$

Elimination of $t$ from (1) gives

$$
x^{p / \epsilon} y^{q / \epsilon}+y^{p / \epsilon_{z}^{q / \epsilon}}+z^{p / \epsilon} x^{q / \epsilon}=0 .
$$

Hence the curves may be projected by a real projection so as to have trigonal symmetry, as in the case of the cubic. Points with parameters $t, 1 /(1-t),(t-1) / t$ are those related by the symmetry. If $p=q, p$ and $q$ are factors of $\epsilon$, and the curve is one of the "triangular-symmetric" curves discussed elsewhere. $\ddagger$ We shall therefore suppose $p$ and $q$ unequal in

* For each non-singular or acnodal cubic, two such real triangles exist. $\dagger$ † See Hilton, Plane Algebraic Curves, Clarendon Press, p. 148. This book is referred to later as " H. P. A. C."

$\ddagger$ Messenger of Mathematics, vol. 50, (1921), p. 171. 
what follows, and there is no loss of generality in supposing $p>q$. The methods of page 138 of H.P.A. C. show that the curve has no cusps other than those involved in the singularities at $A, B, C$; and that the only inflexions other than those at $A, B, C$ are the three real inflexions given by

$$
p q(p-q)\left(t^{3}-3 t+1\right)=\left\{\left(p^{2}-p q+q^{2}\right)(p+q-1)\right.
$$

The tangent at the point (1) is $\left.-3 p q^{2}\right\}\left(t^{2}-t\right)$.

$$
\begin{aligned}
& t^{p}\{-p t+(p-q)\} x+(-1)^{p}(t-1)^{p-q}\{(p-q) t \\
& +q\} y+(-1)^{q} t^{p-q}(t-1)^{p}(q t-p) z=0 .
\end{aligned}
$$

From each of the points $A, B, C$ one real tangent can be drawn other than the sides of the triangle $A B C$. The parameters of the points of contact are $(p-q) / q, q /(q-p), p / q$.

2. Nodes. Consider now the parameters $t_{1}, t_{2}$ of a node of the curve other than $A, B, C$. We have

$$
\left(\frac{t_{1}-1}{t_{2}-1}\right)^{p}=\left(\frac{t_{1}}{t_{2}}\right)^{p}\left(\frac{t_{1}-1}{t_{2}-1}\right)^{q}=\left(\frac{t_{1}}{t_{2}}\right)^{q} \text {. }
$$

Eliminating first $\left(t_{1}-1\right) /\left(t_{2}-1\right)$ and then $t_{1} / t_{2}$ from (6), we find that both these quantities are roots of the algebraic equation $x^{\epsilon}=1$. We write therefore

(7) $t_{2}=\omega t_{1}, \quad\left(t_{2}-1\right)=\Omega\left(t_{1}-1\right), \quad$ where $\quad \omega^{\epsilon}=\Omega^{\epsilon}=1$.

Then (6) and (7) combined give

$$
(1-\Omega) /(\omega-\Omega) \quad \text { and } \quad \varphi(1-\Omega) /(\omega-\Omega)
$$

for the parameters of the node, subject to

$$
\omega^{\epsilon}=\Omega^{\epsilon}=1, \quad \Omega^{p}=\omega^{q}, \quad \Omega^{q} \omega^{p-q}=1 .
$$

We now proceed to find the solutions of (9).

Let $d$ be the highest common factor of $p$ and $q$, and put

$$
p=d p^{\prime}, \quad q=d q^{\prime},
$$

where $p^{\prime}, q^{\prime}$ are prime to one another. We have

$$
\omega=e^{2 k \pi i / \epsilon}, \quad \Omega=e^{2 K \pi i / \epsilon},
$$

where $k, K$ are any unequal integers included among $1,2,3$, $\cdots, \epsilon-1$, consistent with (9). Now (9) gives

$$
\left\{\begin{array}{l}
k q^{\prime}-K p^{\prime} \equiv 0(\bmod \epsilon / d) \\
k\left(p^{\prime}-q^{\prime}\right)+K q^{\prime} \equiv 0(\bmod \epsilon / d) .
\end{array}\right.
$$


Elimination first of $k$ and then of $K$ from (12) shows that $k$ and $K$ are multiples of $d$. Put

$$
k=d k^{\prime}, \quad K=d K^{\prime} .
$$

Then the integers $k^{\prime}, K^{\prime}$ take all possible values $1,2,3, \cdots$, $\boldsymbol{\epsilon} / d-1$, consistent with

$$
k^{\prime} q^{\prime}-K^{\prime} p^{\prime} \equiv 0\left(\bmod \epsilon / d^{2}\right)
$$

excluding the cases in which $k^{\prime}$ and $K^{\prime}$ are equal and multiples of $\epsilon / d^{2}$. Each value of $k^{\prime}$ which is not a multiple of $\boldsymbol{\epsilon} / d^{2}$ gives $d$ values of $K^{\prime}$, and each value of $k^{\prime}$ which is a multiple of $\epsilon / d^{2}$ gives $d-2$ values of $K^{\prime}$. Hence the number of possible values of $\omega$ and $\Omega$ is

$$
d^{2}\left(\epsilon / d^{2}-1\right)+(d-2)(d-1)=\epsilon-3 d+2 .
$$

Now the replacing of $\omega$ and $\Omega$ by $1 / \omega$ and $1 / \Omega$, respectively, merely interchanges $t_{1}$ and $t_{2}$. Hence the number of nodes other than $A, B, C$ is $\frac{1}{2}(\epsilon-3 d+2)$. If we replace $\omega, \Omega$ by $1 / \Omega, \omega / \Omega$ we get a node obtained by the symmetry of the curve from that given by (7).

The coordinates of the nodes are got on replacing $t$ in (1) by $e^{-k \pi i / \epsilon} \sin K \pi \epsilon \csc [(K-k) \pi / \epsilon]$, and $t-1$ by $e^{-k \pi i / \epsilon} \sin k \pi \epsilon$ $X \csc [(K-k) \pi / \epsilon]$, where $k$ and $K$ are multiples of $d$, such that

$$
k q / d^{2}-K p / d^{2} \equiv 0\left(\bmod \epsilon / d^{2}\right) ;
$$

$d$ being the highest common factor of $p$ and $q$, and $\epsilon$ being $p^{2}-p q+q^{2}$, as stated earlier. We readily see that the value of $t$ is complex and that the ratios of the coordinates are real. Hence the point-singularities of the curve, excluding $A, B, C$, are $\frac{1}{2}(\epsilon-3 d+2)$ real acnodes.

3. The Rationalized Equation. The equations $\$ 1$ (1) give the degree $n$ as $p+q$, and (5) gives the class $m$ as $2 p-q+1$ $(p>q)$. Moreover, the curve is unicursal. Therefore Plücker's equations give, with the notation of page $112 \mathrm{of} \mathrm{H.P.} \mathrm{A.} \mathrm{C.,}$

$$
\begin{gathered}
n=p+q, \quad m=2 p-q+1, \\
\delta=\frac{1}{2}\left(p^{2}+2 p q+q^{2}-3 p-9 q+8\right), \quad \kappa=3(q-1), \\
T=\frac{1}{2}\left(4 p^{2}-4 p q+q^{2}-8 p+7 q\right), \quad \iota=3(p-q) .
\end{gathered}
$$

If we require the equation of the curve in rational form, we may proceed as follows. First suppose $d=1$, so that $p$ is 
prime to $q$. Then from (3) the rationalized equation is

$$
\prod\left(\Omega^{p} x^{p / \epsilon} y^{q / \epsilon}+y^{p / \epsilon} z^{q / \epsilon}+\Omega^{q} z^{p / \epsilon} x^{q / \epsilon}\right)=0,
$$

where $\Omega$ is any root of $x^{\epsilon}=1$. The equation is therefore obtained by equating to zero the circulant determinant, whose first row has $y^{p / \epsilon} z^{q / \epsilon}, z^{p / \epsilon} x^{q / \epsilon}, x^{p / \epsilon} y^{q / \epsilon}$ as first, $(q+1)$ th, $(p+1)$ th elements, the other elements being zero.

By Newton's diagram, or otherwise, we see that the singularity at $A, B$, or $C$ is of the same nature as the origin in the curve with Cartesian equation

$$
0=\left(y^{q}-x^{p}\right)+\sum h x^{\alpha} y^{\beta},
$$

$h$ being a constant, and $\alpha, \beta$ zero or positive integers such that $\alpha q+\beta p>p q$. The expansion of $y$ in terms of $x$ near the origin is of the form (see H. P. A. C., Chap. VI, § 1)

$$
y=x^{p / q}\left(a+b x^{1 / q}+c x^{2 / q}+\cdots\right) .
$$

Now suppose $d \neq 1$. First obtain the rationalized equation $f(x, y, z)=0$ of the curve in which $p$ and $q$ are replaced by $p^{\prime}$ and $q^{\prime}$. Then the required equation is

$$
\prod f\left(\omega_{1} x^{1 / d}, \omega_{2} y^{1 / d}, z^{1 / d}\right)=0 \text {, }
$$

where $\omega_{1}$ and $\omega_{2}$ are any roots of $x^{d}=1$.

It is readily seen that the singularity at $A, B, C$ is of the same nature as the origin in

$$
0=\left(y^{q^{\prime}}-x^{p^{\prime}}\right)^{d}+\sum h x^{\alpha} y^{\beta}
$$

while the expansion of $y$ near the origin is still of the form given in (17). The method of page 84 of H. P. A. C. shows that the polar reciprocal of the singularity is the singularity we get, if we replace $q$ by $p-q$.

By $\S 2$, the point-singularities other than $A, B, C$ are merely $\frac{1}{2}(\epsilon-3 d+2)$ acnodes. Hence, by (15), the singularities at $A, B, C$ are each equivalent, so far as Plücker's equations are concerned, to $\frac{1}{2}(p q-p-3 q+d+2)$ nodes and $(q-1)$ cusps, and therefore to $\frac{1}{2}\left(p^{2}-p q-4 p+3 q+d+2\right)$ bitangents and $(p-q-1)$ inflexions. There are besides $\frac{1}{2}(\epsilon+4 p$ $-2 q-3 d-6)$ other bitangents and 3 other inflexions. This statement may be verified by transforming (19) repeatedly by the substitution of $x y$ for $y$ or $x$, to analyze the singularity at the origin by the method of page 134 of H. P. A. C. 
If we transform (3) by the quadratic transformation which consists in replacing $x, y, z$ by $1 / x, 1 / y, 1 / z$, we get a curve of the same type, but with $p-q$ replacing $q$. We may thus derive properties of one curve from those of the other, or duplicate properties of the same curve, if $p=2 q$. For instance, we see that there are exactly three conics through $A, B, C$ which osculate the curve at a point other than $A, B, C$.

When the curve is projected so as to have trigonal symmetry about a point $O$, the circular points $I, J$ lie on the curve unless $p+q$ is a multiple of 3 . The parameters of $I$ and $J$ are $-\omega$ and $-\omega^{2}$, where $\omega^{3}=1$. If $p+q-1$ is a multiple of $3, I J$ is the tangent at $I$ and $J$. If $p+q-2$ is a multiple of $3, O I$ and $O J$ are the tangents at $I$ and $J$. If $p+q$ is a multiple of $3, t=-\omega$ and $-\omega^{2}$ give the point $O$, which is one of the acnodes. The general shape of the curve may readily be found by tracing roughly the curve with Cartesian parametral equations $x=(t-1)^{p} / t^{q}, y=t^{p-q}(t-1)^{q}$, into which it may be projected. There are four fundamentally different types, according as $p$ and $q$ are odd or even.

4. Special Cases. Some special cases may be noted.

If $p=2$ and $q=1, p=3$ and $q=1$, or $p=q$, the curve can be projected to have the symmetry of the equilateral triangle. Otherwise it can only have trigonal symmetry. In the first two cases there is a second triangle bearing the same relation to the curve as $A B C$.

If $p=2, q=1$, there is one acnode at $(1,1,1)$, and three collinear inflexions given by $t=2 \cos 2 \pi / 9,2 \cos 8 \pi / 9$, $2 \cos 14 \pi / 9$. The points of contact of tangents from $B$ and $C$, other than sides of the triangle $A B C$, are collinear with $A$, while $A, B, C$ are "coincidence points." The points of contact of conics through $A, B, C$ osculating the curve lie on another conic through $A, B, C$. Similar results hold for a non-singular cubic (H. P. A. C., p. 260, Ex. 15); but they do not hold for other values of $p$ and $q$.

If $p=3, q=1$, there are three acnodes $A^{\prime}, B^{\prime}, C^{\prime}$. Remembering the symmetry of the curve, we see that, if $A^{\prime} B^{\prime} C^{\prime}$ is taken as triangle of reference, the equation of the curve 
can be put into the form

$$
y^{2} z^{2}+z^{2} x^{2}+x^{2} y^{2}+2 f x y z(x+y+z)=0 .
$$

To obtain the constant $f$, we notice that the conic through the inflexions and the conic touching the inflexional tangents (H. P. A. C., p. 275) have the property that triangles can be inscribed in the first and circumscribed to the second. This gives $f=-3 / 4$. The two conics are $\left(x^{2}+y^{2}+z^{2}\right)=2(y z$ $+z x+x y), 5\left(x^{2}+y^{2}+z^{2}\right)=6(y z+z x+x y)$. The conic through the inflexions touches the sides of the triangle $A^{\prime} B^{\prime} C^{\prime}$.

We can prove readily that, conversely, any real trinodal quartic, whose inflexions-conic touches the three lines joining the nodes, can be projected into

$$
2\left(y^{2} z^{2}+z^{2} x^{2}+x^{2} y^{2}\right)=3 x y z(x+y+z) .
$$

We may also notice the case of the unicursal quintic with three ordinary cusps such that each cuspidal tangent meets the curve in another cusp, and the case of the unicursal sextic with three rhamphoid cusps. The three osculating conics through the cusps of the quintic touch at collinear points.

5. Further Generalizations. We may generalize our problem still further, and consider the unicursal curve in which a triangle $A B C$ is inscribed, so that $A, B, C$ are each given by a single value of the parameter, while the intersections of $B C, C A, A B$ with the curve lie respectively $a$ at $C$ and $n-a$ at $B, b$ at $A$ and $n-b$ at $C, c$ at $B$ and $n-c$ at $A$.

The equations (1), (2), (3) of $\S 1$ are replaced by

$$
\begin{gathered}
x: y: z=(t-1)^{a}:(-t)^{b}(t-1)^{n-b}:(-t)^{n-c}, \\
\epsilon \equiv n^{2}-n(a+b+c)+(b c+c a+a b), \\
y^{a / \epsilon} z^{(n-a) / \epsilon}+z^{b / \epsilon} x^{(n-b) / \epsilon}+x^{c / \epsilon} y^{(n-c) / \epsilon}=0 .
\end{gathered}
$$

It is still true that there are three inflexions other than $A, B, C$, and that from each of $A, B, C$ a single tangent can be drawn other than the sides of the triangle $A B C$.

The parameters of the nodes other than $A, B, C$ are given by $\S 2(3), \S 2$ (4) being replaced by

$$
\omega^{\epsilon}=\Omega^{\epsilon}=1, \quad \Omega^{a}=\omega^{n-c}, \quad \Omega^{n-b} \omega^{b+c-n}=1 .
$$

We must postpone a more detailed discussion.

Bedford College, Regents Park, London 